\title{
Acute kidney injury due to bilateral urolithiasis in pregnancy: a case report
}

\author{
Vineet Mishra $^{1}{ }^{*},{\text { Bhumika } \operatorname{Vyas}^{1}, \text { Priyankur Roy }^{1} \text {, Shaheen Hokabaj }}^{1}$, Khushali Gandhi ${ }^{1}$, \\ Suwa Ram Saini
}

\author{
${ }^{1}$ Department of Obstetrics and Gynecology, Institute of Kidney Disease and Research Centre, Asarwa, Ahmedabad, \\ Gujarat, India \\ ${ }^{2}$ Department of Obstetrics and Gynecology, S. P. Medical College, Bikaner, Rajasthan, India
}

Received: 22 September 2016

Accepted: 18 October 2016

\section{*Correspondence:}

Dr. Vineet Mishra,

E-mail: vineet.mishra.ikdrc@gmail.com

Copyright: (C) the author(s), publisher and licensee Medip Academy. This is an open-access article distributed under the terms of the Creative Commons Attribution Non-Commercial License, which permits unrestricted non-commercial use, distribution, and reproduction in any medium, provided the original work is properly cited.

\begin{abstract}
Kidney stones are very common and unfortunately do not spare the pregnant population. Anatomical and pathophysiological changes occur in the pregnant females that alter the risk for development of urolithiasis. Acute renal colic during pregnancy is associated with significant potential risks to both mother and fetus. Diagnosis is often challenging because good imaging options without radiation use are limited. Management of diagnosed urolithiasis is unique in the pregnant population and requires multi-disciplinary care. Herein, we report a case of pregnancy which occurred in a state of pre-existing bilateral renal calculi with compromised renal function which subsequently developed into acute kidney injury, and requiring definitive management in the form of PCNL after termination of pregnancy.
\end{abstract}

Keywords: Acute kidney injury, PCNL, Termination of pregnancy, Urolithiasis

\section{INTRODUCTION}

Renal diseases in pregnancy present unique challenges. The prevalence of urolithiasis in general population is high, affecting about $10-15 \%$ of population. Due to its high incidence, the correlation of pregnancy with renal calculi is frequent, especially in the "stone belt" of India, which occupies parts of Maharashtra, Gujarat, Punjab, Haryana, Delhi, and Rajasthan. In these regions, the disease is so prevalent that most of the members of a family usually suffer from kidney stones at some point in their lifetime.

The incidence of urolithiasis in pregnancy is $0.03-0.06 \%$ which is same as that of a non-pregnant female of childbearing age. ${ }^{1}$ The incidence of symptomatic stones during pregnancy is 1 in 224 to 1 in 2000, and symptoms are commoner in second and third trimesters of pregnancy. After a review of 14 series published over a 50-year period, Hendricks et al. reported an average incidence of symptomatic nephrolithiasis to be 1 in 1240 pregnancies $(0.08 \%))^{2}$ The incidence seems to be rising in the last decade which may be attributed to the developing imaging methods and more frequent use of imaging as well as to dietary habits, changing climate conditions, increasing obesity, and diabetes mellitus (DM). ${ }^{3,4}$ The incidence of ureteric stones is up to 1 in 1500 pregnancies and $80-90 \%$ of these cases occur during the second and third trimesters. Ureteric stones are more common in multiparous women; most of these women do not have a prior history of urolithiasis.

Asymptomatic calculi during pregnancy does not require any treatment but symptomatic stones requires vigilance as they can cause pyonephrosis, sometimes causing acute kidney injury (AKI), septicemia, and increases the rate of fetal complications due to premature rupture of membranes and preterm labour by 1.4 to 2.4 times. $^{5,6}$ With conservative management, about $70-80 \%$ of stones pass spontaneously, may be due to dilated urinary tract 
during pregnancy. Rest of the cases require further investigations and management. Very rarely pregnancy complicated with calculi requires termination of pregnancy for preserving maternal renal function. Early diagnosis and timely management can prevent maternal and fetal morbidity and mortality.

\section{CASE REPORT}

A 21 years old female, from Barmer, Rajasthan; gravida 2 para 1, was referred to our tertiary care hospital at 12 weeks pregnancy with bilateral multiple renal calculi with acute kidney injury (AKI). She complained of flank pain, dysuria, high grade fever with chills and rigor for duration of 15 days. Her pain was accompanied with vomiting, and initially was relieved by analgesics but had increased in intensity since the last few days.

She delivered her first child, a healthy male baby by full term caesarean section 1 year back. She had conceived during the period of her locational amenorrhea. She had a prior episode of flank pain 3 years back due to right renal calculi. She was managed conservatively then, and had passed stone in urine after which she did not undergo any follow-up. On referral, she already had altered renal function (serum creatinine- $2.6 \mathrm{md} / \mathrm{dl}$ ).

She was admitted owing to her altered renal function and investigated thoroughly. She was having moderate anaemia (Hb- 8.8gm/dl) with leukocytosis (TLC$33,000 /$ cumm) and elevated serum creatinine $(2.43 \mathrm{mg} / \mathrm{dl})$. Her coagulation profile, electrolytes, thyroid status and urine analysis were within normal limits. Antenatal ultrasonography was performed and it showed single live intrauterine fetus of 13 weeks gestation. Ultrasonography of KUB was also performed and it revealed right kidney of size $10.7 \times 4.1 \mathrm{~cm}$, moderate hydronephrosis, with multiple calculi in the calyx- largest being 20mm; and left kidney of size $12.8 \times 6.1 \mathrm{~cm}$, moderate to gross hydronephrosis, hydroureter $(11 \mathrm{~mm}$ up to iliac vessels crossing) and multiple calculi in middle and lower cortex. She was diagnosed to be a case of 13 week pregnancy with AKI due to multiple renal calculi.

A multidisciplinary approach was taken and reference from urologist and nephrologist was obtained before deciding further treatment plan. In view of otherwise stable general condition, preserved urine output and pregnant status; the nephrologist denied need of any form of renal replacement therapy in form of dialysis. She was started on broad spectrum antibiotics (cefoperazone plus sulbactum) for 7 days followed by plan for long term prophylaxis with Tab. Nitrofurantoin. Urologist planned for Bilateral Double J stenting to relieve obstruction and provide chances for improvement of renal function.

Correction of anemia was done with transfusion of one unit of packed cell volume. Bilateral Double J stenting with 5 French catheters was done under short general anaesthesia. She was kept under observation for improvement of renal function. Post procedure, she showed signs of improvement and serum creatinine levels started falling. Her investigations during her stay were as follows:

Table 1: Blood parameters during hospital stay.

\begin{tabular}{|lllll|} 
& Day 2 & Day 3 (on the day of stenting) & $4^{\text {th }}$ day post stenting & $8^{\text {th }}$ day post stenting \\
\hline S. Creatinine & $2.45 \mathrm{mg} / \mathrm{dl}$ & $3.07 \mathrm{mg} / \mathrm{dl}$ & $2.43 \mathrm{mg} / \mathrm{dl}$ & $2.24 \mathrm{mg} / \mathrm{dl}$ \\
\hline S. Na+ & $135.4 \mathrm{mEq} / \mathrm{L}$ & $132.9 \mathrm{mEq} / \mathrm{L}$ & $131.17 \mathrm{mEq} / \mathrm{L}$ & $132.2 \mathrm{mEq} / \mathrm{L}$ \\
\hline S.K+ & $3.31 \mathrm{mEq} / \mathrm{L}$ & $4.06 \mathrm{mEq} / \mathrm{L}$ & $3.92 \mathrm{mEq} / \mathrm{dl}$ & $3.92 \mathrm{mEq} / \mathrm{L}$ \\
\hline PTH & $51.85 \mathrm{pg} / \mathrm{ml}$ & & & \\
\hline S. Urea & & & $53 \mathrm{mg} / \mathrm{dl}$ \\
\hline
\end{tabular}

Suddenly after 7 days she complained of fever with acute colic pain. Her investigations revealed a rise in creatinine levels $(2.98 \mathrm{mg} / \mathrm{dl})$, urine routine microscopy showed plenty of pus cells. Due to persistent symptoms and rising creatinine levels, definitive management after consultation with urologists was to be planned. In presence of acute infection and fever with multiple calculi, ureteroscopic stone removal was contraindicated. So decision for termination of pregnancy for salvaging renal function and definitive treatment in form of per cutaneous nephrolithotomy was planned. The patient and her relatives were counselled regarding the risks and benefits and written informed consent for medical termination of pregnancy was obtained. The process of termination of pregnancy was initiated by induction with misoprostol $400 \mathrm{mcg}$ per vaginally every $4^{\text {th }}$ hourly and she aborted a 50 grams abortus uneventfully.

After termination of pregnancy, she underwent noncontrast computed tomography (NCCT), for exact localization of calculi. As per NCCT, right kidney seemed to be less functional with multiple calculi in UC, $\mathrm{MC}$ and LC with hydronephrosis and hydroureter (Figure 1) whereas the left kidney revealed multiple calculi in UC and MC with hydronephrosis (Figure 2). Hence, she was posted for left PCNL (to salvage the better functioning kidney first) and change of the right sided Double $\mathrm{J}$ catheter. Following left PCNL, her renal function 
improved (serum creatinine $1.86 \mathrm{mg} / \mathrm{dl}$ on day 5 post-op PCNL). Stone analysis was done which showed presence of calcium oxalate stones (Figure 3) which are less common during pregnancy. She was discharged and was called for follow up after 4 weeks for planning PCNL on right side.

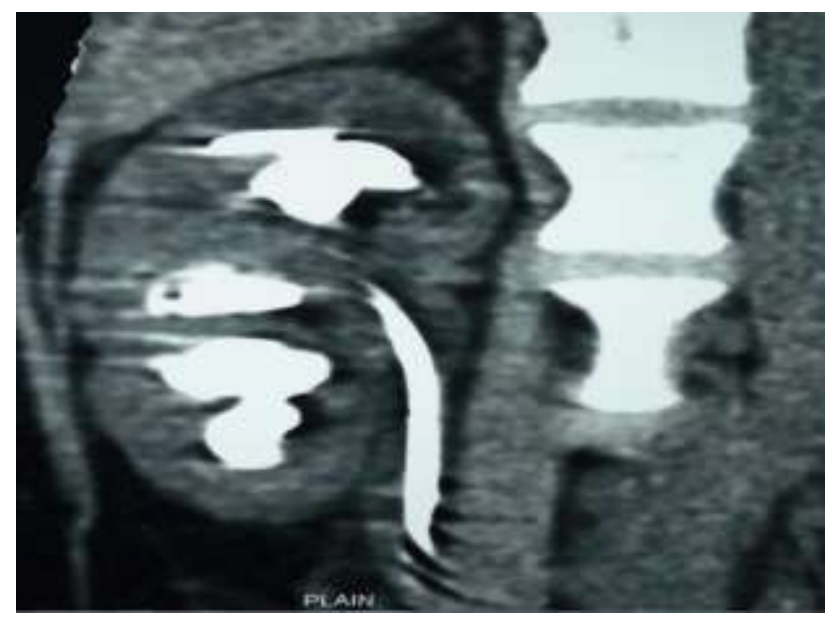

Figure 1: NCCT of right kidney showing multiple calculi in UC, MC and LC with $\mathrm{HN}$ and $\mathrm{HU}$ with DJ stent insitu.

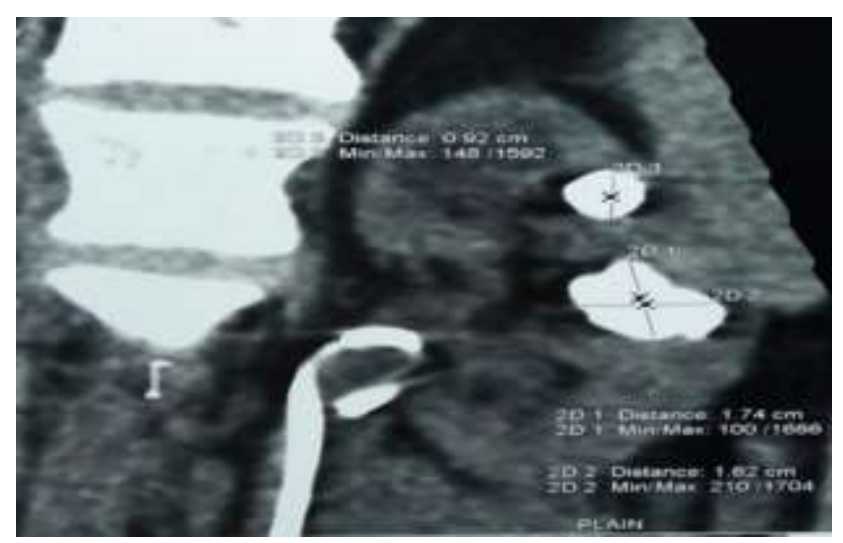

Figure 2: NCCT of left kidney showing multiple calculi in UC and MC with $\mathrm{HN}$ with DJ insitu.

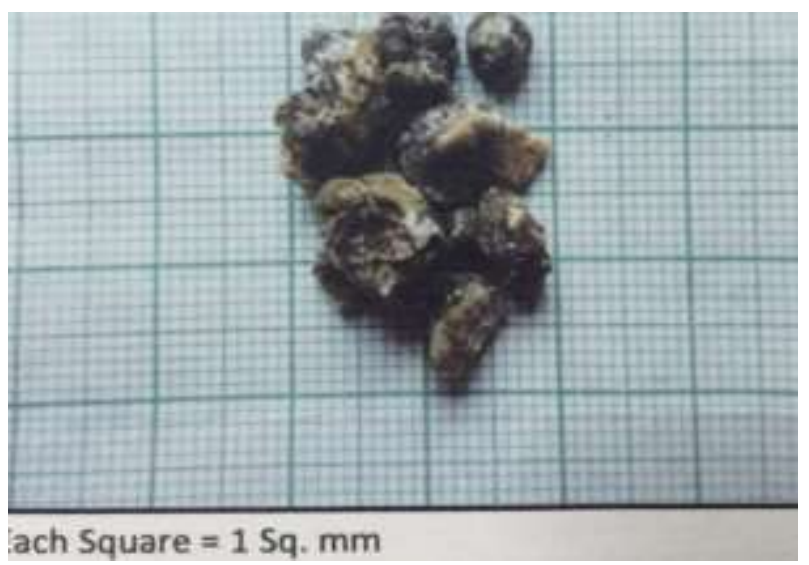

Figure 3: Specimen of calculi.
She was lost to follow up for 4 months and subsequently she returned with complaints of intermittent renal colic since one month which could be explained due to the presence of hydronephrosis on both sides accompanied by colic caused by peristaltic movement for clearance of obstruction caused due to ureteric calculi. Her investigations revealed a rise in serum creatinine levels $(3.49 \mathrm{mg} / \mathrm{dl})$ and urinary infection $(E$. coli - sensitive to Piperacillin Tazobactum). Ultrasonography of KUB was performed and it showed moderate $\mathrm{HN}$ with upper ureteric diameter of $6.5 \mathrm{~mm}$, and multiple calculi in all calyx, largest $15 \mathrm{~mm}$ in the right kidney (Figure 4) while the left kidney revealed minimal $\mathrm{HN}$, upper ureteric diameter of $5 \mathrm{~mm}$, and no calculi.

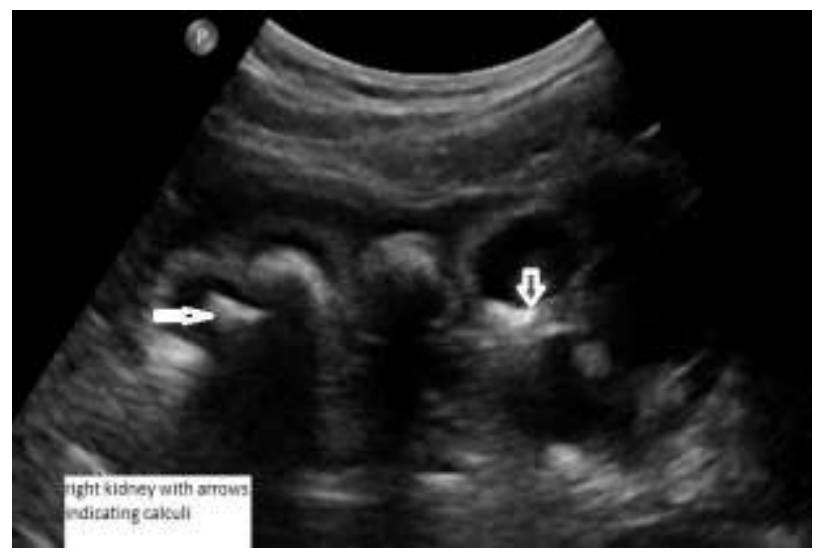

Figure 4: USG of right kidney showing $\mathrm{HN}$ and multiple calculi.

She was readmitted and started on injectable piperacillin tazobactum. She was planned for removal of right double J catheter followed by PCNL. PCNL on right side was carried out in two sittings one day apart. After procedure a temporary nephrostomy tube was kept for 2 days. She was subsequently discharged with serum creatinine levels of $2.12 \mathrm{mg} / \mathrm{dl}$. Though she appeared to have apparently recovered but she had persistent permanent renal damage and was asked for close follow-up with nephrologist.

\section{DISCUSSION}

Pregnancy is characterized by varied physiological changes which lead to marked alteration in renal functions. The physiological elevation in maternal urinary calcium excretion and $\mathrm{pH}$ are likely to have a role in the preferential formation of calcium phosphate stones during pregnancy. ${ }^{7,8}$ Risk factors for stone formation are hereditary factors, age (third to fifth decade of life), decreased water intake, increased environmental temperature, diet (rich in calcium, sodium and meat consumption) and excessive weight or obesity. Urolithiasis associated with ureteral obstruction and upper urinary tract infection is a true urologic emergency and mandates immediate treatment to prevent urosepsis, perinephric abscess formation or even death. 
The incidence of AKI remains high in developing countries with high mortality rate of about more than $50 \%$. In India, the incidence varies from $4-15 \% .^{9}$ Data from our institute, states it to be $9.06 \%$ with most common cause being sepsis followed by hemorrhage and preeclampsia. ${ }^{10}$ AKI in pregnancy can be pre-renal, renal or post-renal. Generally increment in serum creatinine of at least $0.5 \mathrm{mg} / \mathrm{dl}$, in urea nitrogen of $10 \mathrm{mg} / \mathrm{dl}$ or a reduction in urine output below $400 \mathrm{ml} / 24$ hour is considered diagnostic criteria for acute renal failure. AKI in pregnancy causes acid-base and electrolyte imbalance, volume overload, risk of infections, preterm labour and delivery in mother whereas fetal risks are prematurity and dehydration after birth due to osmotic diuresis. The clinical course of AKI is usually that of acute tubular necrosis, but rarely may it result in patchy or complete cortical necrosis, which may lead to residual permanent renal damage which over the years may progress to chronic renal disease and make patient dependant on some form of renal replacement therapy.

Urolithiasis in pregnancy may initiate premature labour or interfere with the progression of normal labour, which poses a significant health risk to fetus. With prompt evaluation and early treatment, both maternal and fetal prognoses are excellent. Very rarely, pregnancy complicated with calculi requires termination of pregnancy for preserving maternal renal function. The primary mode of management should be the conservative. Ureteric stones become symptomatic mostly in the midtrimester which necessitates an intervention. ${ }^{11}$ In conservative approach, patients must be closely monitored with physical examination, total blood count, renal function test and ultrasonography. Urine should be cultured and appropriate antibiotic as per sensitivity should be administered.

Effective and rapid ureteral decompression by the retrograde (via cystoscopy) or antegrade (after percutaneous renal puncture) insertion of an indwelling ureteral Double-J stent can be used in pregnant women with obstructed renal system. DJ stent must be changed every 4-6 weeks as it can get blocked and bacterial colonization can occur. Percutaneous nephrostomy is effectively used under local anaesthesia with 98-100\% success rate in obstructed systems. Patients with an indwelling nephrostomy tube may proceed to PCNL after delivery, but PCNL is not advised during pregnancy. Although case reports have described successful PCNL in early pregnancy, the necessity for general anaesthesia need for fluoroscopy and prone position of the patient make this a hazardous procedure. ${ }^{12}$ Important advantages of PCNL over DJ stent are the absence of lower urinary tract complaints and also it provides access for definitive treatment later.

Postpartum follow up after 3 months is recommended to eliminate confusion in interpreting findings as ureteral and calyceal dilatation may persist that long. Best measure is prevention as specific therapy for stones can be offered only after pregnancy and unresolvable or persistent stones may require termination of pregnancy if reported in first or early $2^{\text {nd }}$ trimester. Patients with history of renal stones should be evaluated before pregnancy for recurrent UTI.

Our patient differed from other patients as her pregnancy occurred in a state of pre-existing renal calculi. This shows that pregnancy occurring in pre-existing calculi can further aggravate compromised renal function. She had bilateral renal calculi with compromised renal function, with fever and acute urinary tract infection with AKI, which required definitive management in the form of PCNL after termination of pregnancy.

\section{CONCLUSION}

Renal calculi during pregnancy should be treated promptly and a serious follow-up should be advised. A patient with history of renal calculi should undergo screening and treatment for the same during preconceptional period. Rarely, when pregnancy is complicated with multiple calculi with kidney damage, termination of pregnancy and definitive management should be considered. No evidence in favour of restriction of calcium supplementation during pregnancy is available and same should not be restricted, rather in patient with stone forming tendency urinary acidifying agents with plenty of fluid intake and dietary modification should be done.

A tough decision for treating obstetrician after discussion with the patient and her relatives should be taken. In our case, the decision was in favour of termination of pregnancy, on basis of preventing permanent maternal renal impairment, which, in spite of all efforts, could not be completely prevented.

\section{Funding: No funding sources \\ Conflict of interest: None declared \\ Ethical approval: Not required}

\section{REFERENCES}

1. Butler EL, Cox SM, Eberts EG, Cunningham FG. Symptomatic nephrolithiasis complicating pregnancy. Obstet Gynecol. 2000;96:753.

2. Hendricks SK, Ross SO, Krieger JN. An algorithm for diagnosis and therapy of management and complications of urolithiasis during pregnancy. Surg Gynecol Obstet. 1991;172:49-54.

3. Asplin JR. Obesity and urolithiasis. Adv Chronic Kidney Dis. 2009;16:11-20.

4. Scales CD, Smith AC, Hanley JM. Urologic diseases in America, Project. Prevalence of kidney stones in the United States. Eur Urol. 2012;62:160-5.

5. Lewis DF, Robichaux AG, Jaekle RK. Urolithiasis in pregnancy. Diagnosis, management and pregnancy outcome. J Reprod Med. 2003;48:28-32. 
6. Eisner BH, Reese A, Sheth S. Ureteral sto $\neg$ ne location at emergency room presentation with colic. J Urol. 2009; 182:165-8.

7. Ross AE, Handa S, Lingeman JE. Kidney stones during pregnancy: An investigation into stone composition. Urol Res. 2008;36:99-102.

8. Skolarikos A, Laguna MP, Alivizatos G. The role for active monitoring in urinary stones: A systematic review. J Endourol. 2010;24:923-30.

9. Chugh KS, Singhal PC, Sharma BK. Acute renal failure of obstetric origin. Obstet Gynecol. 1976;48:642.

10. Goplani KR, Shah PR, Gera DN, Gumber M, Dabhi M, Feroz A, et al. Pregnancy-related acute renal failure: A single-center experience. Indian J Nephrol. 2008;18:17-21.
11. N'gamba M, Lebdai S, Hasting C. Acute renal colic during pregnancy: Management and predictive factors. Can J Urol. 2015;22:7732-8.

12. ACOG Committee on Obstetric Practice. ACOG Committee Opinion. Number 299, September 2004 (replaces No. 158, September 1995). Guidelines for diagnostic imaging during pregnancy. Obstet Gynecol. 2004;104:647-51.

Cite this article as: Mishra V, Vyas B, Roy P, Hokabaj S, Gandhi K, Saini SR. Acute kidney injury due to bilateral urolithiasis in pregnancy: a case report. Int J Reprod Contracept Obstet Gynecol 2016;5:4486-90. 